\title{
Symmetric Bent-core Liquid Crystals of Some Schiff Bases Containing Azo Linkage
}

\author{
Catalina Ionica Ciobanu, Irina Carlescu, Gabriela Lisa, and Dan Scutaru* \\ Department of Natural and Synthetic Polymers, Faculty of Chemical Engineering and Environmental Protection, \\ "Gheorghe Asachi" Technical University of Iasi, Bd. D. Mangeron 71A, 700050 Iasi, Romania
}

RECEIVED JULY 27, 2012; REVISED OCTOBER 14, 2013; ACCEPTED OCTOBER 21, 2013

\begin{abstract}
The paper presents the synthesis and liquid crystalline properties of some new symmetric bentcore liquid crystals containing a 1,3-dihydroxybenzene central core disubstituted with mesogenic units consisting of three aromatic rings connected through esteric, azomethinic and azo groups. The wings are ended with terminal acyloxy or alkyloxy chains containing 6 to 10 carbon atoms. Compounds were obtained by the condensation reactions between some 4-((4-alcanoyloxyphenyl)azo)benzaldehydes or 4-((4alkyloxyphenyl)azo)benzaldehydes with the 1,3-phenylene bis(4-aminobenzoate) core. The new synthesized compounds were characterized from structural point of view $\left({ }^{1} \mathrm{H} N M R,{ }^{13} \mathrm{C}\right.$ NMR, MS spectroscopy) and liquid crystalline properties (polarizing optical microscopy and differential scanning calorimetry). All the banana shaped compounds presented enantiotropic liquid crystalline properties, with a wideranging stability of mesophases both on heating and cooling cycles. The synthesized compounds presented a good thermal stability in the existence range of the mesophases as evidenced by thermogravimetric studies. (doi: 10.5562/cca2150)
\end{abstract}

Keywords: Schiff Bases, symmetric bent-core compounds, mesomorphic properties, azo linkage

\section{INTRODUCTION}

In the last period, bent-core molecules attracted considerable interest because of their capability to generate new mesophases with non-conventional properties. Compared to classical calamitics, structure-property relationships in banana shaped liquid crystals are more difficult to predict. The main characteristic is the nonlinear (bent-shaped) structure achieved by connecting two mesogenic groups to a central core such as 1,3disubstituted benzene, 2,7-disubstituted naphthalene, 2,5-disubstituted [1,3,4]oxadiazole, 2,5-disubstituted thiophene, 2,6-disubstituted pyridine etc. Several hundred of compounds having a bent molecular shape and exhibiting characteristic mesophases have been reported so far. $^{1-6}$

Up to now, eight phases formed by bent-core molecules and designated as $B_{1}-B_{8}$ have been found and most of them present additional sub-phases. ${ }^{7,8}$ The shape of banana achiral mesogens cause steric interactions that led to unique phases of special complexity from columnar $\left(\mathrm{B}_{1}\right)$ to smectic $\left(\mathrm{B}_{2}, \mathrm{~B}_{3}, \mathrm{~B}_{6}\right.$ and $\left.\mathrm{B}_{7}\right)$ up to nematic ordering." These "banana type phases" are specific phases different from conventional phases found in calamitic mesogens. ${ }^{10}$ Among them, the phases
$\mathrm{B}_{1}, \mathrm{~B}_{2}$ and $\mathrm{B}_{6}$ are the most commonly observed on banana shaped compounds. ${ }^{11-14}$ The smectic liquid crystalline phase separates from isotropic liquid in a rich variety of forms, from classical "batonnets" to filamentary shapes or helical coils in lyotropic systems. From these, more frequently are elongated structures consisting of focal-conic domains.

Probably the most interesting mesophases exhibit the bent-core compounds containing the thermally sensitive azomethine group. Among rod-shaped mesogens, the Schiff bases tend to order into smectic phases. Otherwise the first smectic mesophases of banana shaped molecules had azomethine connecting group between aromatic rings. ${ }^{15}$

The first reported bent-core compounds containing azo linkage into structure has been reported since 2001 and contains six aromatic rings. ${ }^{16}$ Since then, some other bent shaped molecules containing azobenzene moieties have been synthesized, the majority of these molecules presenting interesting liquid crystalline properties. ${ }^{16-24}$

The goal of the paper is to extent the classes of bent shaped molecules containing the azo photoisomerisable group. The paper presents the synthesis of some bent-core liquid crystals based on a 1,3-dihydroxy-

\footnotetext{
* Author to whom correspondence should be addressed. (E-mail: dscutaru@ch.tuiasi.ro)
} 
benzene core with two symmetric wings containing each three benzene rings connected via esteric, azomethinic and azo groups. One of the series ends with terminal acyloxy chains while the other one contains alkyloxy ones. The introduction of the $-\mathrm{N}=\mathrm{N}$ - linkage contributes to the extension of conjugation between the aromatic rings and may induce photo-responsive properties due to the capacity to generate trans-cis isomerization under UV-VIS irradiation. All the obtained compounds presented enantiotropic liquid crystalline properties, mainly with smectic type textures.

\section{EXPERIMENTAL}

\section{Materials}

Analytical reagents such as resorcinol, $p$-nitrobenzoic acid, DCC, DMAP, were provided by Aldrich (Germany) or by Merck (Germany) and were used without further purification. Synthesis of $\mathbf{3 a}-\mathbf{3 e}$ and $\mathbf{4 a}-\mathbf{4 e}$ compounds was previously reported. ${ }^{25}$ Silica gel 60 (Merck) was used for column chromatography (CC). The chromatography (TLC) was performed on silica gel plates (Merck, silica gel $\mathrm{F}_{254}$ ).

\section{Instrumentation}

Confirmation of the structures of the intermediates and final products was obtained by ${ }^{1} \mathrm{H}$ NMR and ${ }^{13} \mathrm{C}$ NMR, using a Bruker Avance DRX $400 \mathrm{MHz}$ spectrometer (Rheinstein, Germany) with tetramethylsilane as internal standard. Mass spectra were recorded on a quadrupole time-of-flight mass spectrometer equipped with an electrospray ion source (Agilent 6520 Accurate Mass Q-TOF LC=MS (Santa Clara, CA,USA).

Transition temperatures were determined using a Linkam heating stage and Linksys 32 temperature control unit in conjunction with a Axioscop 40 Zeiss polarizing optical microscope and Qimaging/Retiga-1000R camera for image capture, the transitions being confirmed by DSC analysis (Mettler Toledo DSC1). All thermal analyses were performed on a Mettler-Toledo TGA SDTA $851^{\mathrm{e}}$ derivatograph in an $\mathrm{N}_{2}$ atmosphere, with a flow rate of $20 \mathrm{~mL} \mathrm{m^{-1 }}$ and a heating rate of $10{ }^{\circ} \mathrm{C} \min ^{-1}$ from $25{ }^{\circ} \mathrm{C}$ to $900{ }^{\circ} \mathrm{C}$. In order to obtain comparable data, constant operational parameters were kept for all samples.

\section{Synthesis}

\section{1,3-phenylene bis(4-nitrobenzoate) (1)}

To a solution of $2 \mathrm{~g}(18.18 \mathrm{mmol})$ resorcinol in $30 \mathrm{~mL}$ anhydrous $\mathrm{CH}_{2} \mathrm{Cl}_{2}, 6.07 \mathrm{~g}$ (36.36 mmol) 4-nitrobenzoic acid and a catalytic amount of DMAP as catalyst were added under stirring. Over the mixture a solution of 8.25 g $(40 \mathrm{mmol}) \mathrm{DCC}$ in $50 \mathrm{~mL}$ anhydrous $\mathrm{CH}_{2} \mathrm{Cl}_{2}$ was added. After stirring overnight at room temperature, the diciclohexyl urea was filtered off and the solution was concentrated. The product was purified by column chromatography silicagel/dichloromethane. Yield: $5 \mathrm{~g}$ (62 \%), m.p. $185{ }^{\circ} \mathrm{C} .{ }^{1} \mathrm{H}$ NMR $\left(\mathrm{CDCl}_{3}\right) \delta /$ ppm: $8.38(\mathrm{~m}$, $8 \mathrm{H}, \mathrm{Ar}), 7.55$ (m, 1H, Ar), 7.25 (d, 1H, Ar, $J=1.96 \mathrm{~Hz}$ ), $7.23\left(\mathrm{dd}, 2 \mathrm{H}, \mathrm{Ar}, J_{1}=2.16 \mathrm{~Hz}, J_{2}=2.15 \mathrm{~Hz}\right),{ }^{13} \mathrm{C} \mathrm{NMR}$ (DMSO-d6) $\delta /$ ppm: 162.95, 151.08, 151.05, 134.55, $131.38,130.32,123.84,119.50,115.47(-\mathrm{O}-\mathrm{C}=\mathrm{O}+$ $8 \mathrm{C}$, aromatic).

\section{1,3-phenylene bis(4-aminobenzoate) (2)}

$1 \mathrm{~g}$ (2.45 mmol) 1,3-phenylene bis(4-nitrobenzoate) (1) was dissolved under reflux in $50 \mathrm{~mL}$ anhydrous ethanol and $5.54 \mathrm{~g}$ (12.25 mmol) $\mathrm{SnCl}_{2} \cdot 2 \mathrm{H}_{2} \mathrm{O}$ (as a solid) was added in several portions. The mixture was heated under stirring and reflux for 6 hours. After cooling, the reaction mixture was poured in a beaker containing ice water and treated with a solution of $5 \% \mathrm{NaOH}$ until the $\mathrm{pH}=8$. The fine organic suspension was extracted several times with ethyl acetate, the collected extracts were washed several times with water, dried up with $\mathrm{MgSO}_{4}$ and then concentrated. The purification of the product was made by recrystallization from diethyl ether. Yield: 0.6 g (60 \%), m.p. $192{ }^{\circ} \mathrm{C} .{ }^{1} \mathrm{H}$ NMR (DMSO-d6) $\delta /$ ppm: $7.82(\mathrm{~d}, 4 \mathrm{H}, \mathrm{Ar}, J=8.70 \mathrm{~Hz}), 7.48(\mathrm{~m}, 1 \mathrm{H}, \mathrm{Ar})$, $7.13(\mathrm{~m}, 3 \mathrm{H}, \mathrm{Ar}), 6.66(\mathrm{~d}, 4 \mathrm{H}, \mathrm{Ar}, J=8.71 \mathrm{~Hz}), 6.20$ (s, 4H, 2* $-\mathrm{NH}_{2}$ ), ${ }^{13} \mathrm{C}$ NMR (DMSO-d6) $\delta / \mathrm{ppm}: 164.2$, 154.14, 151.33, 131.81, 129.48, 119.04, 116.14, 114.08, $112.63(-\mathrm{O}-\mathrm{C}=\mathrm{O}+8 \mathrm{C}$, aromatic).

General procedure for the synthesis of the Schiff Bases $5 a-5 e$ and $6 a-6 e$

1,3-Phenylene bis(4-aminobenzoate) (2) and the corresponding 3a-3e or $\mathbf{4 a}-\mathbf{4 e}$ aldehydes were refluxed under stirring, for two hours, in anhydrous ethanol containing a few drops of glacial acetic acid as catalyst. The obtained suspension was filtered while hot and washed twice with ethanol. The resulting products were purified by recrystallization from ethanol.

1,3-phenylene bis(4-(4-((4-hexanoyloxyphenyl)azo)benzylideneamino)benzoate) (5a)

Quantities: $0.25 \mathrm{~g}$ (0.72 mmol) 1,3-phenylene bis(4aminobenzoate) (2) in $15 \mathrm{~mL}$ ethanol, $0.46 \mathrm{~g}$ (1.44 mmol) 4-((4-hexanoyloxyphenyl)azo)benzaldehyde (3a) in $15 \mathrm{~mL}$ ethanol, glacial acetic acid (1-2 drops), yield: $0.35 \mathrm{~g}(51 \%)$, liquid crystal: $169{ }^{\circ} \mathrm{C}\left(\mathrm{K}_{1} / \mathrm{K}_{2}\right), 202{ }^{\circ} \mathrm{C}$ $\left(\mathrm{K}_{2} / \mathrm{LC}\right), 315^{\circ} \mathrm{C}(\mathrm{LC} / \mathrm{I}), 307{ }^{\circ} \mathrm{C}(\mathrm{I} / \mathrm{LC}), 193{ }^{\circ} \mathrm{C}\left(\mathrm{LC} / \mathrm{K}_{1}\right)$, $166{ }^{\circ} \mathrm{C}\left(\mathrm{K}_{1} / \mathrm{K}_{2}\right) .{ }^{1} \mathrm{H} \mathrm{NMR}\left(\mathrm{CDCl}_{3}\right) \delta / \mathrm{ppm}: 8.53(\mathrm{~s}, 2 \mathrm{H}$, $\left.2^{*}-\mathrm{CH}=\mathrm{N}-\right), 8.25$ (d, $\left.4 \mathrm{H}, \mathrm{Ar}, J=8.55 \mathrm{~Hz}\right), 8.08$ (d, $4 \mathrm{H}, \mathrm{Ar}, J=8.33 \mathrm{~Hz}), 8.01$ (d, $4 \mathrm{H}, \mathrm{Ar}, J=8.77 \mathrm{~Hz}), 7.98$ (d, $4 \mathrm{H}, \mathrm{Ar}, J=8.77 \mathrm{~Hz}), 7.48(\mathrm{~m}, 1 \mathrm{H}, \mathrm{Ar}), 7.29(\mathrm{~m}, 9 \mathrm{H}$, Ar), $7.20\left(\mathrm{dd}, 2 \mathrm{H}, \mathrm{Ar}, J_{1}=8.33 \mathrm{~Hz}, J_{2}=8.11 \mathrm{~Hz}\right)$, $2.58\left(\mathrm{t}, 4 \mathrm{H}, 2^{*}-\mathrm{O}-\mathrm{CO}-\mathrm{CH}_{2}-\right), 1.80\left(\mathrm{qv}, 4 \mathrm{H}, 2^{*}-\mathrm{CH}_{2}-\right)$, $1.43\left(\mathrm{~m}, 8 \mathrm{H}, 4 *-\mathrm{CH}_{2}-\right), 0.95\left(\mathrm{t}, 6 \mathrm{H}, 2^{*}-\mathrm{CH}_{3}\right)$, ${ }^{13} \mathrm{C}$ NMR $\left(\mathrm{CDCl}_{3}\right) \delta / \mathrm{ppm}: 171.73,164.46,160.77$, $156.88,154.72,153.53,151.81,150.42,137.87,131.65$, $130.03,128.78,126.99,124.35,123.37,122.33,120.97$, 
119.17, $115.86(2 *-\mathrm{O}-\mathrm{C}=\mathrm{O}+-\mathrm{CH}=\mathrm{N}-+16 \mathrm{C}$, aromatic), 34.51, 31.34, 24.64, 22.30, 13.81 (5C, aliphatic), $\mathrm{m} / z: 959.96[\mathrm{M}]^{+}$.

1,3-phenylene bis(4-(4-((4-heptanoyloxyphenyl)azo) benzylideneamino)benzoate) (5b)

Quantities: $0.25 \mathrm{~g}$ (0.72 mmol) 1,3-phenylene bis(4aminobenzoate) (2) in $15 \mathrm{~mL}$ ethanol, $0.49 \mathrm{~g}(1.44 \mathrm{mmol})$ 4-((4-heptanoyloxyphenyl)azo)benzaldehyde (3b) in 15 $\mathrm{mL}$ ethanol, glacial acetic acid (1-2 drops), yield: $0.33 \mathrm{~g}$ (46.5\%), liquid crystal: $164{ }^{\circ} \mathrm{C}\left(\mathrm{K}_{1} / \mathrm{K}_{2}\right), 209{ }^{\circ} \mathrm{C}\left(\mathrm{K}_{2} /\right.$ LC), $304{ }^{\circ} \mathrm{C}(\mathrm{LC} / \mathrm{I}), 302{ }^{\circ} \mathrm{C}(\mathrm{I} / \mathrm{LC}), 200{ }^{\circ} \mathrm{C}\left(\mathrm{LC} / \mathrm{K}_{1}\right)$, $158{ }^{\circ} \mathrm{C}\left(\mathrm{K}_{1} / \mathrm{K}_{2}\right) .{ }^{1} \mathrm{H} \mathrm{NMR}\left(\mathrm{CDCl}_{3}\right) \delta / \mathrm{ppm}: 8.53$ (s, 2H, $\left.2^{*}-\mathrm{CH}=\mathrm{N}-\right), 8.24(\mathrm{~d}, 4 \mathrm{H}, \mathrm{Ar}, J=8.11 \mathrm{~Hz}), 8.07$ (d, $4 \mathrm{H}, \mathrm{Ar}, J=7.89 \mathrm{~Hz}), 7.99$ (m, 8H, Ar), 7.48 (m, 1H, Ar), 7.29 (m, 9H, Ar), $7.20\left(\mathrm{dd}, 2 \mathrm{H}, \mathrm{Ar}, J_{1}=8.33 \mathrm{~Hz}\right.$, $\left.J_{2}=8.11 \mathrm{~Hz}\right), 2.58\left(\mathrm{t}, 4 \mathrm{H}, 2^{*}-\mathrm{O}-\mathrm{CO}-\mathrm{CH}_{2}-\right), 1.78(\mathrm{qv}$, $\left.4 \mathrm{H}, 2^{*}-\mathrm{CH}_{2}-\right), 1.40\left(\mathrm{~m}, 12 \mathrm{H}, 6^{*}-\mathrm{CH}_{2}-\right), 0.92(\mathrm{t}, 6 \mathrm{H}$, $\left.2 *-\mathrm{CH}_{3}\right),{ }^{13} \mathrm{C}$ NMR $\left(\mathrm{CDCl}_{3}\right) \delta / \mathrm{ppm}: 171.74,164.45$, $160.77,156.84,154.67,153.49,151.78,150.38,137.87$, $131.63,130.02,129.78,126.95,124.34,123.36,122.32$, $120.97,119.16,115.85,(2 *-\mathrm{O}-\mathrm{C}=\mathrm{O}+-\mathrm{CH}=\mathrm{N}-+16$ $\mathrm{C}$, aromatic), 34.53, 31.46, 28.80, 24.92, 22.47, 13.92 (6C, aliphatic), $m / z: 987.72[\mathrm{M}]^{+}$.

1,3-phenylene bis(4-(4-((4-octanoyloxyphenyl)azo)benzylideneamino)benzoate) (5c)

Quantities: $0.25 \mathrm{~g}$ (0.72 mmol) 1,3-phenylene bis(4aminobenzoate) (2) in $15 \mathrm{~mL}$ ethanol, $0.51 \mathrm{~g}$ (1.44 mmol) 4-((4-octanoyloxyphenyl)azo)-benzaldehyde (3c) in $15 \mathrm{~mL}$ ethanol, glacial acetic acid (1-2 drops), yield: $0.38 \mathrm{~g}(52 \%)$, liquid crystal: $146{ }^{\circ} \mathrm{C}\left(\mathrm{K}_{1} / \mathrm{K}_{2}\right), 207{ }^{\circ} \mathrm{C}$ $\left(\mathrm{K}_{2} / \mathrm{LC}\right), 285^{\circ} \mathrm{C}(\mathrm{LC} / \mathrm{I}), 277^{\circ} \mathrm{C}(\mathrm{I} / \mathrm{LC}), 190^{\circ} \mathrm{C}\left(\mathrm{LC} / \mathrm{K}_{1}\right)$. ${ }^{1} \mathrm{H}$ NMR $\left(\mathrm{CDCl}_{3}\right) \delta$ / ppm: 8.52 (s, 2H, 2* $-\mathrm{CH}=\mathrm{N}-), 8.24(\mathrm{~d}, 4 \mathrm{H}, \mathrm{Ar}, J=7.90 \mathrm{~Hz}), 8.07$ (d, 4H, Ar, $J=7.89 \mathrm{~Hz}), 8.00$ (d, 4H, Ar, $J=8.98 \mathrm{~Hz}), 7.98(\mathrm{~d}$, $4 \mathrm{H}, \mathrm{Ar}, J=8.99 \mathrm{~Hz}), 7.47$ (m, 1H, Ar), 7.29 (m, 9H, Ar), $7.19(\mathrm{~d}, 2 \mathrm{H}, \mathrm{Ar}, J=7.90 \mathrm{~Hz}), 2.58\left(\mathrm{t}, 4 \mathrm{H}, 2^{*}\right.$ $-\mathrm{O}-\mathrm{CO}-\mathrm{CH}_{2}-$ ), 1.78 (qv, 4H, 2* $-\mathrm{CH}_{2}-$ ), $1.44(\mathrm{~m}, 16 \mathrm{H}$, $\left.8^{*}-\mathrm{CH}_{2}-\right), 0.91\left(\mathrm{t}, 6 \mathrm{H}, 2 *-\mathrm{CH}_{3}\right),{ }^{13} \mathrm{C} \mathrm{NMR}\left(\mathrm{CDCl}_{3}\right) \delta /$ ppm: 171.74, 164.44, 160.77, 156.84, 154.67, 153.49, $151.78,150.38,137.87,131.63,130.02,129.78,126.94$, $124.34,123.36,122.32,120.97,119.16,115.85,(2 *$ $-\mathrm{O}-\mathrm{C}=\mathrm{O}+-\mathrm{CH}=\mathrm{N}-+16 \mathrm{C}$, aromatic), 34.52, 31.67, 29.11, 28.90, 24.96, 22.58, 13.97 ( 7C, aliphatic), $\mathrm{m} / \mathrm{z}$ : $1015.48[\mathrm{M}-1]^{+}$.

1,3-phenylene bis(4-(4-((4-nonanoyloxyphenyl)azo)benzylideneamino)benzoate) (5d)

Quantities: $0.25 \mathrm{~g}$ (0.72 mmol) 1,3-phenylene bis(4aminobenzoate) (2) in $15 \mathrm{~mL}$ ethanol, $0.53 \mathrm{~g}$ (1.44 mmol) 4-((4-nonanoyloxyphenyl)azo)benzaldehyde (3d) in $15 \mathrm{~mL}$ ethanol, glacial acetic acid (1-2 drops), yield: $0.4 \mathrm{~g}(53 \%)$, liquid crystal: $148{ }^{\circ} \mathrm{C}\left(\mathrm{K}_{1} / \mathrm{K}_{2}\right), 202{ }^{\circ} \mathrm{C}$ $\left(\mathrm{K}_{2} / \mathrm{LC}\right), 288^{\circ} \mathrm{C}(\mathrm{LC} / \mathrm{I}), 285^{\circ} \mathrm{C}(\mathrm{I} / \mathrm{LC}), 190{ }^{\circ} \mathrm{C}\left(\mathrm{LC} / \mathrm{K}_{1}\right)$, $86{ }^{\circ} \mathrm{C}\left(\mathrm{K}_{1} / \mathrm{K}_{2}\right) .{ }^{1} \mathrm{H}$ NMR $\left(\mathrm{CDCl}_{3}\right) \delta / \mathrm{ppm}: 8.52(\mathrm{~s}, 2 \mathrm{H}$, $\left.2^{*}-\mathrm{CH}=\mathrm{N}-\right), 8.24$ (d, 4H, Ar, $\left.J=8.55 \mathrm{~Hz}\right), 8.07$ (d,
4H, Ar, $J=8.55 \mathrm{~Hz}), 7.99$ (m, 8H, Ar), 7.47 (m, 1H, Ar), $7.28(\mathrm{~m}, 9 \mathrm{H}, \mathrm{Ar}), 7.20\left(\mathrm{dd}, 2 \mathrm{H}, \mathrm{Ar}, J_{1}=8.12 \mathrm{~Hz}, J_{2}\right.$ $=8.33 \mathrm{~Hz}), 2.58\left(\mathrm{t}, 4 \mathrm{H}, 2^{*}-\mathrm{O}-\mathrm{CO}-\mathrm{CH}_{2}-\right), 1.78(\mathrm{qv}$, $\left.4 \mathrm{H}, 2 *-\mathrm{CH}_{2}-\right), 1.36\left(\mathrm{~m}, 20 \mathrm{H}, 10 *-\mathrm{CH}_{2}-\right), 0.90(\mathrm{t}, 6 \mathrm{H}$, $\left.2 *-\mathrm{CH}_{3}\right),{ }^{13} \mathrm{C}$ NMR $\left(\mathrm{CDCl}_{3}\right) \delta / \mathrm{ppm}: 171.74,164.44$, $160.75,156.87,154.73,153.54,151.83,150.43,137.91$, 131.64, 130.03, 129.77, 127.00, 124.34, 123.36, 122.32, $120.97,119.15,115.84,(2 *-\mathrm{O}-\mathrm{C}=\mathrm{O}+-\mathrm{CH}=\mathrm{N}-+16$ C, aromatic), 34.55, 31.83, 29.22, 29.17, 29.11, 24.97, 22.62, 13.97 (8C, aliphatic), $m / z: 1044.49\left[\mathrm{M}^{+}\right.$.

1,3-phenylene bis(4-(4-((4-decanoyloxyphenyl)azo)benzylideneamino)benzoate) (5e)

Quantities: $0.25 \mathrm{~g}$ (0.72 mmol) 1,3-phenylene bis(4aminobenzoate) (2) in $15 \mathrm{~mL}$ ethanol, $0.55 \mathrm{~g}$ (1.44 mmol) 4-((4-decanoyloxyphenyl)azo)benzaldehyde (3e) in $15 \mathrm{~mL}$ ethanol, glacial acetic acid (1-2 drops), yield: 0.3 g $(39 \%)$, liquid crystal: $148{ }^{\circ} \mathrm{C}\left(\mathrm{K}_{1} / \mathrm{K}_{2}\right), 195{ }^{\circ} \mathrm{C}$ $\left(\mathrm{K}_{2} / \mathrm{LC}\right), 287^{\circ} \mathrm{C}(\mathrm{LC} / \mathrm{I}), 285^{\circ} \mathrm{C}(\mathrm{I} / \mathrm{LC}), 185^{\circ} \mathrm{C}\left(\mathrm{LC} / \mathrm{K}_{1}\right)$, $178^{\circ} \mathrm{C}\left(\mathrm{K}_{1} / \mathrm{K}_{2}\right) .{ }^{1} \mathrm{H} \mathrm{NMR}\left(\mathrm{CDCl}_{3}\right) \delta / \mathrm{ppm}: 8.53(\mathrm{~s}, 2 \mathrm{H}$, $\left.2^{*}-\mathrm{CH}=\mathrm{N}-\right), 8.25$ (d, $\left.4 \mathrm{H}, \mathrm{Ar}, J=8.33 \mathrm{~Hz}\right), 8.08$ (d, $4 \mathrm{H}, \mathrm{Ar}, J=8.55 \mathrm{~Hz}), 8.01$ (d, $4 \mathrm{H}, \mathrm{Ar}, J=8.77 \mathrm{~Hz}), 7.98$ (d, 4H, Ar, $J=8.77 \mathrm{~Hz}), 7.48(\mathrm{~m}, 1 \mathrm{H}, \mathrm{Ar}), 7.28(\mathrm{~m}, 9 \mathrm{H}$, Ar), $7.20\left(\mathrm{dd}, 2 \mathrm{H}, \mathrm{Ar}, J_{1}=8.20 \mathrm{~Hz}, J_{2}=2 \mathrm{~Hz}\right), 2.58(\mathrm{t}$, $\left.4 \mathrm{H}, 2 *-\mathrm{O}-\mathrm{CO}-\mathrm{CH}_{2}-\right), 1.78\left(\mathrm{qv}, 4 \mathrm{H}, 2^{*}-\mathrm{CH}_{2}-\right), 1.39$ (m, 24H, 12*- $\left.\mathrm{CH}_{2}-\right), 0.89$ (t, 6H, 2* $\left.-\mathrm{CH}_{3}\right),{ }^{13} \mathrm{C} \mathrm{NMR}$ $\left(\mathrm{CDCl}_{3}\right) \delta /$ ppm: 171.76, 164.45, 160.79, 156.85, 154.66, $153.49,151.76,150.37,137.86,131.64,130.02,129.79$, 126.93, 124.34, 123.37, 122.32, 120.97, 119.18, 115.85, (2* $-\mathrm{O}-\mathrm{C}=\mathrm{O}+-\mathrm{CH}=\mathrm{N}-+16 \mathrm{C}$, aromatic), 34.52, $31.88,29.43,29.27,29.25,29.15,24.96,22.65,14.02$ (9C, aliphatic), $m / z: 1071.01[\mathrm{M}-1]^{+}$.

1,3-phenylene bis(4-(4-((4-hexyloxyphenyl)azo)benzylideneamino)benzoate) (6a)

Quantities: $0.25 \mathrm{~g}$ (0.72 mmol) 1,3-phenylene bis(4aminobenzoate) (2) in $15 \mathrm{~mL}$ ethanol, $0.44 \mathrm{~g}$ (1.44 mmol) 4-((4-hexyloxyphenyl)azo)benzaldehyde (4a) in $15 \mathrm{~mL}$ ethanol, glacial acetic acid (1-2 drops), yield: $0.41 \mathrm{~g}(61.2 \%)$, liquid crystal: $159{ }^{\circ} \mathrm{C}\left(\mathrm{K}_{1} / \mathrm{K}_{2}\right), 181{ }^{\circ} \mathrm{C}$ $\left(\mathrm{K}_{2} / \mathrm{LC}_{1}\right), 215{ }^{\circ} \mathrm{C}\left(\mathrm{LC}_{1} / \mathrm{LC}_{2}\right), 294{ }^{\circ} \mathrm{C}\left(\mathrm{LC}_{2} / \mathrm{I}\right), 293{ }^{\circ} \mathrm{C}$ $\left(\mathrm{I} / \mathrm{LC}_{1}\right), 202{ }^{\circ} \mathrm{C}\left(\mathrm{LC}_{1} / \mathrm{LC}_{2}\right), 197{ }^{\circ} \mathrm{C}\left(\mathrm{LC}_{2} / \mathrm{K}_{1}\right), 149{ }^{\circ} \mathrm{C}$ $\left(\mathrm{K}_{1} / \mathrm{K}_{2}\right) .{ }^{1} \mathrm{H}$ NMR $\left(\mathrm{CDCl}_{3}\right) \delta / \mathrm{ppm}: 8.52\left(\mathrm{~s}, 2 \mathrm{H}, 2^{*}\right.$ $-\mathrm{CH}=\mathrm{N}-), 8.24(\mathrm{~d}, 4 \mathrm{H}, \mathrm{Ar}, J=8.33 \mathrm{~Hz}), 8.05(\mathrm{~d}, 4 \mathrm{H}$, Ar, $J=8.33 \mathrm{~Hz}), 7.97$ (d, 4H, Ar, $J=8.55 \mathrm{~Hz}), 7.94(\mathrm{~d}$, $4 \mathrm{H}, \mathrm{Ar}, J=8.99 \mathrm{~Hz}), 7.47$ (m, 1H, Ar), 7.29 (d, 4H, Ar, $J=8.55 \mathrm{~Hz}), 7.24$ (m, 1H, Ar), 7.19 (dd, 2H, Ar, $J_{1}=$ $\left.8.12 \mathrm{~Hz}, J_{2}=8.11 \mathrm{~Hz}\right), 7.01(\mathrm{~d}, 4 \mathrm{H}, \mathrm{Ar}, J=8.99 \mathrm{~Hz})$, $4.06\left(\mathrm{t}, 4 \mathrm{H}, 2 *-\mathrm{O}-\mathrm{CH}_{2}-\right), 1.83\left(\mathrm{qv}, 4 \mathrm{H}, 2 *-\mathrm{CH}_{2}-\right.$ ), $1.50\left(\mathrm{qv}, 4 \mathrm{H}, 2^{*}-\mathrm{CH}_{2}-\right), 1.36\left(\mathrm{~m}, 8 \mathrm{H}, 4^{*}-\mathrm{CH}_{2}\right), 0.92$ $\left(\mathrm{t}, 6 \mathrm{H}, 2^{*}-\mathrm{CH}_{3}\right) .{ }^{13} \mathrm{C} \mathrm{NMR}\left(\mathrm{CDCl}_{3}\right) \delta / \mathrm{ppm}: 164.47$, $162.44,160.94,157.01,155.06,151.82,147.28,137.23$, $131.64,130.01,129.76,126.88,125.18,123.08,120.97$, $119.16,115.85,115.01,(-\mathrm{O}-\mathrm{C}=\mathrm{O}+-\mathrm{CH}=\mathrm{N}-+16 \mathrm{C}$, aromatic), $68.64\left(-\mathrm{O}-\mathrm{CH}_{2}\right), 31.60,29.25,25.73,22.58$, 13.91 (5C, aliphatic), $m / z: 932.30[\mathrm{M}]^{+}$. 
1,3-phenylene bis(4-(4-((4-heptyloxyphenyl)azo)benzylideneamino)benzoate) (6b)

Quantities: $0.25 \mathrm{~g}$ (0.72 mmol) 1,3-phenylene bis(4aminobenzoate) (2) in $15 \mathrm{~mL}$ ethanol, $0.47 \mathrm{~g}$ (1.44 mmol) 4-((4-heptyloxyphenyl)azo)benzaldehyde (4b) in $15 \mathrm{~mL}$ ethanol, glacial acetic acid (1-2 drops), yield: $0.37 \mathrm{~g}(53.6 \%)$, liquid crystal: $151{ }^{\circ} \mathrm{C}\left(\mathrm{K}_{1} / \mathrm{K}_{2}\right), 181{ }^{\circ} \mathrm{C}$ $\left(\mathrm{K}_{2} / \mathrm{LC}_{1}\right), 213{ }^{\circ} \mathrm{C}\left(\mathrm{LC}_{1} / \mathrm{LC}_{2}\right), 278{ }^{\circ} \mathrm{C}\left(\mathrm{LC}_{2} / \mathrm{I}\right), 274{ }^{\circ} \mathrm{C}$ (I/LC), $201{ }^{\circ} \mathrm{C}\left(\mathrm{LC} / \mathrm{K}_{1}\right), 145{ }^{\circ} \mathrm{C}\left(\mathrm{K}_{1} / \mathrm{K}_{2}\right) .{ }^{1} \mathrm{H}$ NMR $\left(\mathrm{CDCl}_{3}\right) \delta /$ ppm: 8.52 (s, 2H, 2* $\left.-\mathrm{CH}=\mathrm{N}-\right), 8.24(\mathrm{~d}, 4 \mathrm{H}$, Ar, $J=8.33 \mathrm{~Hz}), 8.05$ (d, 4H, Ar, $J=8.33 \mathrm{~Hz}), 7.97$ (d, $4 \mathrm{H}, \mathrm{Ar}, J=8.55 \mathrm{~Hz}), 7.94$ (d, 4H, Ar, $J=8.99 \mathrm{~Hz}), 7.48$ (m, 1H, Ar), 7.30 (d, 4H, Ar, $J=8.33 \mathrm{~Hz}), 7.24(\mathrm{~m}, 1 \mathrm{H}$, Ar), $7.20\left(\mathrm{dd}, 2 \mathrm{H}, \mathrm{Ar}, J_{1}=8.30 \mathrm{~Hz}, J_{2}=2.20 \mathrm{~Hz}\right), 7.01$ (d, 4H, Ar, $J=8.99 \mathrm{~Hz}), 4.06\left(\mathrm{t}, 4 \mathrm{H}, 2 *-\mathrm{O}-\mathrm{CH}_{2}-\right.$ ), $1.83\left(\mathrm{qv}, 4 \mathrm{H}, 2^{*}-\mathrm{CH}_{2}-\right), 1.49\left(\mathrm{qv}, 4 \mathrm{H}, 2^{*}-\mathrm{CH}_{2}-\right), 1.30$ $\left(\mathrm{m}, 12 \mathrm{H}, 6 *-\mathrm{CH}_{2}\right), 0.91\left(\mathrm{t}, 6 \mathrm{H}, 2^{*}-\mathrm{CH}_{3}\right),{ }^{13} \mathrm{C} \mathrm{NMR}$ $\left(\mathrm{CDCl}_{3}\right) \delta$ / ppm: 164.49, 162.45, 160.94, 157.01, 155.07, $151.82,147.27,137.23,131.63,130.01,129.77,126.87$, $125.18,123.08,120.98,119.16,115.87,115.02$, (-O$\mathrm{C}=\mathrm{O}+-\mathrm{CH}=\mathrm{N}-+16 \mathrm{C}$, aromatic $), 68.65\left(-\mathrm{O}-\mathrm{CH}_{2}\right)$, 31.80, 29.29, 29.05, 26.04, 22.59, 13.97 (6C, aliphatic), $\mathrm{m} / \mathrm{z}: 960.05[\mathrm{M}]^{+}$.

1,3-phenylene bis(4-(4-((4-octyloxyphenyl)azo)benzylideneamino)benzoate) (6c)

Quantities: $0.25 \mathrm{~g}$ (0.72 mmol) 1,3-phenylene bis(4aminobenzoate) (2) in $15 \mathrm{~mL}$ ethanol, $0.49 \mathrm{~g}(1.44$ mmol) 4-((4-octyloxyphenyl)azo)benzaldehyde (4c) in $15 \mathrm{~mL}$ ethanol, glacial acetic acid (1-2 drops), yield: $0.31 \mathrm{~g}(43.6 \%)$, liquid crystal: $135^{\circ} \mathrm{C}\left(\mathrm{K}_{1} / \mathrm{K}_{2}\right), 201{ }^{\circ} \mathrm{C}$ $\left(\mathrm{K}_{2} / \mathrm{LC}\right), 267^{\circ} \mathrm{C}(\mathrm{LC} / \mathrm{I}), 263{ }^{\circ} \mathrm{C}(\mathrm{I} / \mathrm{LC}), 185^{\circ} \mathrm{C}\left(\mathrm{LC} / \mathrm{K}_{1}\right)$, $130{ }^{\circ} \mathrm{C}\left(\mathrm{K}_{1} / \mathrm{K}_{2}\right) .{ }^{1} \mathrm{H} \mathrm{NMR}\left(\mathrm{CDCl}_{3}\right) \delta / \mathrm{ppm}: 8.51(\mathrm{~s}, 2 \mathrm{H}$, $\left.2^{*}-\mathrm{CH}=\mathrm{N}-\right), 8.24(\mathrm{~d}, 4 \mathrm{H}, \mathrm{Ar}, J=8.33 \mathrm{~Hz}), 8.05$ (d, $4 \mathrm{H}, \mathrm{Ar}, J=8.33 \mathrm{~Hz}), 7.97$ (d, 4H, Ar, $J=8.55 \mathrm{~Hz}), 7.94$ (d, 4H, Ar, $J=8.99 \mathrm{~Hz}), 7.47$ (m, 1H, Ar), 7.29 (d, 4H, Ar, $J=8.55 \mathrm{~Hz}), 7.24(\mathrm{~m}, 1 \mathrm{H}, \mathrm{Ar}), 7.19$ (dd, 2H, Ar, $J_{1}$ $\left.=8.12 \mathrm{~Hz}, J_{2}=8.33 \mathrm{~Hz}\right), 7.01(\mathrm{~d}, 4 \mathrm{H}, \mathrm{Ar}, J=8.99 \mathrm{~Hz})$, $4.06\left(\mathrm{t}, 4 \mathrm{H}, 2 *-\mathrm{O}-\mathrm{CH}_{2}-\right), 1.83\left(\mathrm{qv}, 4 \mathrm{H}, 2 *-\mathrm{CH}_{2}-\right.$ ), 1.49 (qv, 4H, 2* $\left.-\mathrm{CH}_{2}-\right), 1.33\left(\mathrm{~m}, 16 \mathrm{H}, 8^{*}-\mathrm{CH}_{2}\right), 0.91$ $\left(\mathrm{t}, 6 \mathrm{H}, 2^{*}-\mathrm{CH}_{3}\right),{ }^{13} \mathrm{C} \mathrm{NMR}\left(\mathrm{CDCl}_{3}\right) \delta / \mathrm{ppm}: 164.48$, $162.44,160.93,156.99,155.06,151.81,147.26,137.21$, 131.63, 130.01, 129.77, 126.86, 125.17, 123.07, 120.98, $119.16,115.86,115.01(-\mathrm{O}-\mathrm{C}=\mathrm{O}+-\mathrm{CH}=\mathrm{N}-+16 \mathrm{C}$, aromatic), $68.64\left(-\mathrm{O}-\mathrm{CH}_{2}\right), 31.83,29.36,29.27,29.22$, 26.08, 22.64, 13.99 (7C, aliphatic), $m / z$ : $988.79[\mathrm{M}]^{+}$.

1,3-phenylene bis(4-(4-((4-nonyloxyphenyl)azo)benzylideneamino)benzoate) (6d)

Quantities: $0.25 \mathrm{~g}(0.72 \mathrm{mmol})$ 1,3-phenylene bis(4aminobenzoate) (2) in $15 \mathrm{~mL}$ ethanol, $0.5 \mathrm{~g}(1.44 \mathrm{mmol})$ 4-((4-nonyloxyphenyl)azo)benzaldehyde (4d) in $15 \mathrm{~mL}$ ethanol, glacial acetic acid (1-2 drops), yield: $0.38 \mathrm{~g}$ (52\%), liquid crystal: $195{ }^{\circ} \mathrm{C}(\mathrm{K} / \mathrm{LC}), 260{ }^{\circ} \mathrm{C}(\mathrm{LC} / \mathrm{I})$, $257{ }^{\circ} \mathrm{C}(\mathrm{I} / \mathrm{LC}), 169{ }^{\circ} \mathrm{C}(\mathrm{LC} / \mathrm{K}) .{ }^{1} \mathrm{H}$ NMR $\left(\mathrm{CDCl}_{3}\right) \delta /$ ppm: $8.52\left(\mathrm{~s}, 2 \mathrm{H}, 2^{*}-\mathrm{CH}=\mathrm{N}-\right), 8.24(\mathrm{~d}, 4 \mathrm{H}, \mathrm{Ar}, J=$
$8.33 \mathrm{~Hz}), 8.05$ (d, 4H, Ar, $J=8.33 \mathrm{~Hz}), 7.97$ (d, 4H, Ar, $J=8.33 \mathrm{~Hz}), 7.94(\mathrm{~d}, 4 \mathrm{H}, \mathrm{Ar}, J=8.77 \mathrm{~Hz}), 7.48(\mathrm{~m}$, $1 \mathrm{H}, \mathrm{Ar}), 7.30$ (d, 4H, Ar, $J=8.55 \mathrm{~Hz}), 7.24(\mathrm{~m}, 1 \mathrm{H}$, Ar), 7.19 (dd, $\left.2 \mathrm{H}, \mathrm{Ar}, J_{1}=8.12 \mathrm{~Hz}, J_{2}=8.11 \mathrm{~Hz}\right), 7.01$ (d, $4 \mathrm{H}, \mathrm{Ar}, J=8.99 \mathrm{~Hz}), 4.06\left(\mathrm{t}, 4 \mathrm{H}, 2 *-\mathrm{O}-\mathrm{CH}_{2}-\right.$ ), 1.83 (qv, 4H, 2*- $\mathrm{CH}_{2}-$ ), 1.49 (qv, 4H, 2* $\left.-\mathrm{CH}_{2}-\right), 1.34$ $\left(\mathrm{m}, 20 \mathrm{H}, 10^{*}-\mathrm{CH}_{2}\right), 0.89\left(\mathrm{t}, 6 \mathrm{H}, 2^{*}-\mathrm{CH}_{3}\right),{ }^{13} \mathrm{C} \mathrm{NMR}$ $\left(\mathrm{CDCl}_{3}\right) \delta$ / ppm: 164.49, 162.44, 160.95, 156.99, 155.05, $151.80,147.25,137.21,131.63,130.01,129.78,126.85$, $125.17,123.07,120.98,119.17,115.87,115.01$ (-O$\mathrm{C}=\mathrm{O}+-\mathrm{CH}=\mathrm{N}-+16 \mathrm{C}$, aromatic $), 68.63\left(-\mathrm{O}-\mathrm{CH}_{2}\right)$, $31.90,29.54,29.40,29.28,29.25,26.07,22.66,14.00$ (8C, aliphatic), $m / z: 1016.58[\mathrm{M}]^{+}$.

1,3-phenylene bis(4-(4-((4-decyloxyphenyl)azo)benzylideneamino)benzoate) (6e)

Quantities: 0.25 g (0.72 mmol) 1,3-phenylene bis(4aminobenzoate) (2) in $15 \mathrm{~mL}$ ethanol, $0.52 \mathrm{~g}$ (1.44 mmol) 4-((4-decyloxyphenyl)azo)benzaldehyde (4e) in $15 \mathrm{~mL}$ ethanol, glacial acetic acid (1-2 drops), yield: $0.31 \mathrm{~g}(41.3 \%)$, liquid crystal: $152{ }^{\circ} \mathrm{C}\left(\mathrm{K}_{1} / \mathrm{K}_{2}\right), 177^{\circ} \mathrm{C}$ $\left(\mathrm{K}_{2} / \mathrm{LC}\right), 259^{\circ} \mathrm{C}(\mathrm{LC} / \mathrm{I}), 257^{\circ} \mathrm{C}(\mathrm{I} / \mathrm{LC}), 166^{\circ} \mathrm{C}\left(\mathrm{LC} / \mathrm{K}_{1}\right)$, $149{ }^{\circ} \mathrm{C}\left(\mathrm{K}_{1} / \mathrm{K}_{2}\right) .{ }^{1} \mathrm{H} \mathrm{NMR}\left(\mathrm{CDCl}_{3}\right) \delta / \mathrm{ppm}: 8.52(\mathrm{~s}, 2 \mathrm{H}$, $\left.2^{*}-\mathrm{CH}=\mathrm{N}-\right), 8.24$ (d, 4H, Ar, $\left.J=8.33 \mathrm{~Hz}\right), 8.06$ (d, $4 \mathrm{H}, \mathrm{Ar}, J=8.33 \mathrm{~Hz}), 7.97$ (d, 4H, Ar, $J=8.33 \mathrm{~Hz}), 7.94$ (d, $4 \mathrm{H}, \mathrm{Ar}, J=8.77 \mathrm{~Hz}), 7.48$ (m, 1H, Ar), 7.30 (d, 4H, $\mathrm{Ar}, J=8.33 \mathrm{~Hz}), 7.25$ (m, 1H, Ar), 7.19 (dd, 2H, Ar, $J_{1}=$ $\left.8.12 \mathrm{~Hz}, J_{2}=8.11 \mathrm{~Hz}\right), 7.01(\mathrm{~d}, 4 \mathrm{H}, \mathrm{Ar}, J=8.77 \mathrm{~Hz})$, $4.06\left(\mathrm{t}, 4 \mathrm{H}, 2^{*}-\mathrm{O}-\mathrm{CH}_{2}-\right), 1.83\left(\mathrm{qv}, 4 \mathrm{H}, 2^{*}-\mathrm{CH}_{2}-\right)$, 1.49 (qv, 4H, 2* $\left.-\mathrm{CH}_{2}-\right), 1.35\left(\mathrm{~m}, 24 \mathrm{H}, 12 *-\mathrm{CH}_{2}\right)$, $0.89\left(\mathrm{t}, 6 \mathrm{H}, 2^{*}-\mathrm{CH}_{3}\right) .{ }^{13} \mathrm{C} \mathrm{NMR}\left(\mathrm{CDCl}_{3}\right) \delta / \mathrm{ppm}$ : 164.48, 162.43, 160.96, 156.98, 155.03, 151.77, 147.22, $137.19,131.63,130.01,129.78,126.83,125.17,123.07$, $120.98,119.17,115.87,114.99(-\mathrm{O}-\mathrm{C}=\mathrm{O}+-\mathrm{CH}=\mathrm{N}-+$ $16 \mathrm{C}$, aromatic), $68.62\left(-\mathrm{O}-\mathrm{CH}_{2}\right), 31.92,29.58,29.56$, 29.40, 29.31, 29.27, 26.07, 22.67, 14.02 (9C, aliphatic), $m / z: 1043.32[\mathrm{M}-1]^{+}$.

\section{RESULTS AND DISCUSSION}

\section{Synthesis}

The bent-core Schiff Bases were obtained by the biscondensation reactions between the 1,3-phenylene bis(4-aminobenzoate) core (2) and the corresponding mesogenic aldehydes $\mathbf{3 a}-\mathbf{3 e}$ or $\mathbf{4 a - 4 e}$ (Scheme 1). Compounds $\mathbf{2}$ and $\mathbf{3 a}-\mathbf{3 e}$ or $\mathbf{4 a}-\mathbf{4 e}$ were refluxed in anhydrous ethanol in the presence of glacial acetic acid as catalyst. The core compound $\mathbf{2}$ was obtained in two steps. In the first step, resorcinol was reacted with 4-nitrobenzoic acid in the presence of DCC / DMAP. The obtained 1,3-phenylene bis(4-nitrobenzoate) (1) was reduced to 1,3-phenylene bis(4-aminobenzoate) (2) by adding in several portions solid $\mathrm{SnCl}_{2} \cdot 2 \mathrm{H}_{2} \mathrm{O}$. Compounds 3a-3e were synthesized by treating 4-((4hydroxyphenyl)azo)benzaldehyde with the correspond- 
<smiles>[R]Oc1ccc(N=Nc2ccc(C=Nc3ccc(C(=O)Oc4cccc(OC(=O)c5ccc(C=Nc6ccc(N=Nc7ccc([R20])cc7)cc6)cc5)c4)cc3)cc2)cc1</smiles>

Scheme 1. Synthesis of the Schiff bases based on a 1,3-phenylene bis(4-aminobenzoate) core

ing $n$-aliphatic carboxylic acids in the presence of DCC and DMAP. ${ }^{14}$ The synthesis of intermediates $\mathbf{4 a - 4 e}$ involved the Williamson etherification of 4-((4-hydroxyphenyl)azo)benzaldehyde with the corresponding $n$-alkyl bromides in ethanol in the presence of $\mathrm{KOH}^{5}$

\section{Liquid Crystalline Properties}

Polarized optical microscopy (POM) and differential scanning calorimetry (DSC) investigations evidenced for all the synthesized compounds enantiotropic liquid crystalline properties, mainly with different smectic or nematic textures. The domains of the existence of the mesophases are quite wide for both $5 \mathbf{a}-5 \mathbf{e}$ and $\mathbf{6 a - 6 e}$ classes (between 63 and $114{ }^{\circ} \mathrm{C}$, Table 1). Thermogravimetric studies evidenced a good thermal stability for all the obtained compounds, in all the cases the $T_{\text {onset }}$ values being superior to the isotropisation temperatures (Table 1.).

The 5a-5e Schiff bases contain an esteric group in the terminal chain. DSC investigations evidenced for all the 5a-5e compounds three transitions, both on the heating and cooling cycles. Figure 1a presents, as an example, the DSC curves of compound 5e.

In the case of the first two compounds of the series, $\mathbf{5} \mathbf{a}$ and $\mathbf{5 b}$, the $T_{\text {onset }}$ values are very close to the clearing points and Table 1 presents the isotropisation temperatures observed during the POM investigations. For compound 5a the mesophase started on heating at $202{ }^{\circ} \mathrm{C}$ with a smectic like texture which maintained up to isotropisation at $315^{\circ} \mathrm{C}$ (Figure 2a). Upon cooling from the isotropic liquid, a nematic phase appeared for a wide temperature interval of about $78^{\circ} \mathrm{C}$ with characteristic Schlieren nematic texture (Figure 2b). On further cooling, the Schlieren texture transformed into a smectic one that maintained down to $193{ }^{\circ} \mathrm{C}$ when crystallization occurred.

Although the compound $\mathbf{5 b}$ presented similar values of the temperature transitions as $\mathbf{5 a}$, it has not showed nematic textures on cooling, only smectic ordering being evidenced for an interval of about $100{ }^{\circ} \mathrm{C}$ (Figure 2c). 
Table 1. Transition temperatures (expressed in ${ }^{\circ} \mathrm{C}$ ) and transition enthalpies (expressed in $\mathrm{Jg}^{-1}$ ) for compounds $5 \mathbf{a}-\mathbf{e}$ and $\mathbf{6 a}-\mathbf{e}$

\begin{tabular}{|c|c|c|c|c|c|c|c|c|c|c|}
\hline \multicolumn{2}{|c|}{ Compound } & \multirow{2}{*}{$\begin{array}{c}\mathrm{K}_{1} / \mathrm{K}_{2} \\
169 \\
{[-3]}\end{array}$} & \multirow{2}{*}{$\begin{array}{c}\mathrm{K}_{2} / \mathrm{K}_{3} \\
-\end{array}$} & \multirow{2}{*}{$\begin{array}{c}\mathrm{K}_{3} / \mathrm{LC} \\
202 \\
{[-13]}\end{array}$} & \multirow{2}{*}{$\begin{array}{r}\mathrm{LC} / \mathrm{I} \\
315^{(\mathrm{b})}\end{array}$} & \multirow{2}{*}{$\begin{array}{r}\mathrm{I} / \mathrm{LC} \\
307^{(\mathrm{b})}\end{array}$} & \multirow{2}{*}{$\begin{array}{c}\mathrm{LC}_{1} / \mathrm{LC}_{2} \\
-\end{array}$} & \multirow{2}{*}{$\begin{array}{c}\mathrm{LC}_{2} / \mathrm{K}_{1} \\
193 \\
{[14]}\end{array}$} & \multirow{2}{*}{$\begin{array}{c}\mathrm{K}_{1} / \mathrm{K}_{2} \\
166 \\
{[2]}\end{array}$} & \multirow{2}{*}{$\frac{T_{\text {onset }}^{(\mathrm{a})} /{ }^{\circ} \mathrm{C}}{324}$} \\
\hline $5 a$ & $n=5$ & & & & & & & & & \\
\hline $5 b$ & $n=6$ & $\begin{array}{c}164 \\
{[-2]}\end{array}$ & - & $\begin{array}{c}209 \\
{[-13]}\end{array}$ & $304^{(\mathrm{b})}$ & $302^{(\mathrm{b})}$ & - & $\begin{array}{l}200 \\
{[17]}\end{array}$ & $\begin{array}{l}158 \\
{[3]}\end{array}$ & 329 \\
\hline $5 c$ & $n=7$ & $\begin{array}{c}146 \\
{[-30]}\end{array}$ & - & $\begin{array}{c}207 \\
{[-14]}\end{array}$ & $\begin{array}{c}285 \\
{[-20]}\end{array}$ & $\begin{array}{l}277 \\
{[12]}\end{array}$ & - & $\begin{array}{c}190 \\
{[14]}\end{array}$ & - & 328 \\
\hline $5 d$ & $n=8$ & $\begin{array}{c}148 \\
{[-29]}\end{array}$ & - & $\begin{array}{c}202 \\
{[-17]}\end{array}$ & $\begin{array}{c}288 \\
{[-20]}\end{array}$ & $\begin{array}{l}285 \\
{[19]}\end{array}$ & - & $\begin{array}{l}190 \\
{[17]}\end{array}$ & $\begin{array}{c}86 \\
{[29]}\end{array}$ & 325 \\
\hline $5 e$ & $n=9$ & $\begin{array}{c}148 \\
{[-27]}\end{array}$ & - & $\begin{array}{c}195 \\
{[-17]}\end{array}$ & $\begin{array}{c}287 \\
{[-18]}\end{array}$ & $\begin{array}{l}285 \\
{[18]}\end{array}$ & - & $\begin{array}{c}185 \\
{[16]}\end{array}$ & $\begin{array}{c}78 \\
{[24]}\end{array}$ & 329 \\
\hline $6 a$ & $n=6$ & $\begin{array}{l}159 \\
{[-5]}\end{array}$ & $\begin{array}{l}181 \\
{[-4]}\end{array}$ & $\begin{array}{c}215 \\
{[-15]}\end{array}$ & $\begin{array}{c}294 \\
{[-17]}\end{array}$ & $\begin{array}{l}293 \\
{[17]}\end{array}$ & $\begin{array}{l}202 \\
{[15]}\end{array}$ & $\begin{array}{c}197 \\
{[0.5]}\end{array}$ & $\begin{array}{l}149 \\
{[11]}\end{array}$ & 335 \\
\hline $6 b$ & $n=7$ & $\begin{array}{l}151 \\
{[-5]}\end{array}$ & $\begin{array}{c}181 \\
{[-6]}\end{array}$ & $\begin{array}{c}213 \\
{[-18]}\end{array}$ & $\begin{array}{c}276 \\
{[-17]}\end{array}$ & $\begin{array}{c}274 \\
{[17]}\end{array}$ & - & $\begin{array}{l}201 \\
{[18]}\end{array}$ & $\begin{array}{l}145 \\
{[6]}\end{array}$ & 350 \\
\hline $6 c$ & $n=8$ & $\begin{array}{c}136 \\
{[-0.8]}\end{array}$ & - & $\begin{array}{c}202 \\
{[-16]}\end{array}$ & $\begin{array}{c}267 \\
{[-19]}\end{array}$ & $\begin{array}{l}263 \\
{[15]}\end{array}$ & - & $\begin{array}{c}185 \\
{[15]}\end{array}$ & $\begin{array}{l}130 \\
{[1]}\end{array}$ & 348 \\
\hline $6 d$ & $n=9$ & - & - & $\begin{array}{c}195 \\
{[-20]}\end{array}$ & $\begin{array}{c}260 \\
{[-22]}\end{array}$ & $\begin{array}{l}257 \\
{[22]}\end{array}$ & - & $\begin{array}{l}169 \\
{[20]}\end{array}$ & - & 355 \\
\hline $6 e$ & $n=10$ & $\begin{array}{l}152 \\
{[-1]}\end{array}$ & - & $\begin{array}{c}177 \\
{[-21]}\end{array}$ & $\begin{array}{c}259 \\
{[-20]}\end{array}$ & $\begin{array}{l}257 \\
{[20]}\end{array}$ & - & $\begin{array}{l}166 \\
{[21]}\end{array}$ & $\begin{array}{c}149 \\
{[0.8]}\end{array}$ & 345 \\
\hline
\end{tabular}

(a) $T_{\text {onset }}-$ temperature at which thermal degradation begins.

(b) Data obtained from POM investigations.

Abbreviations: K-crystalline, LC-liquid crystal, I-isotropic
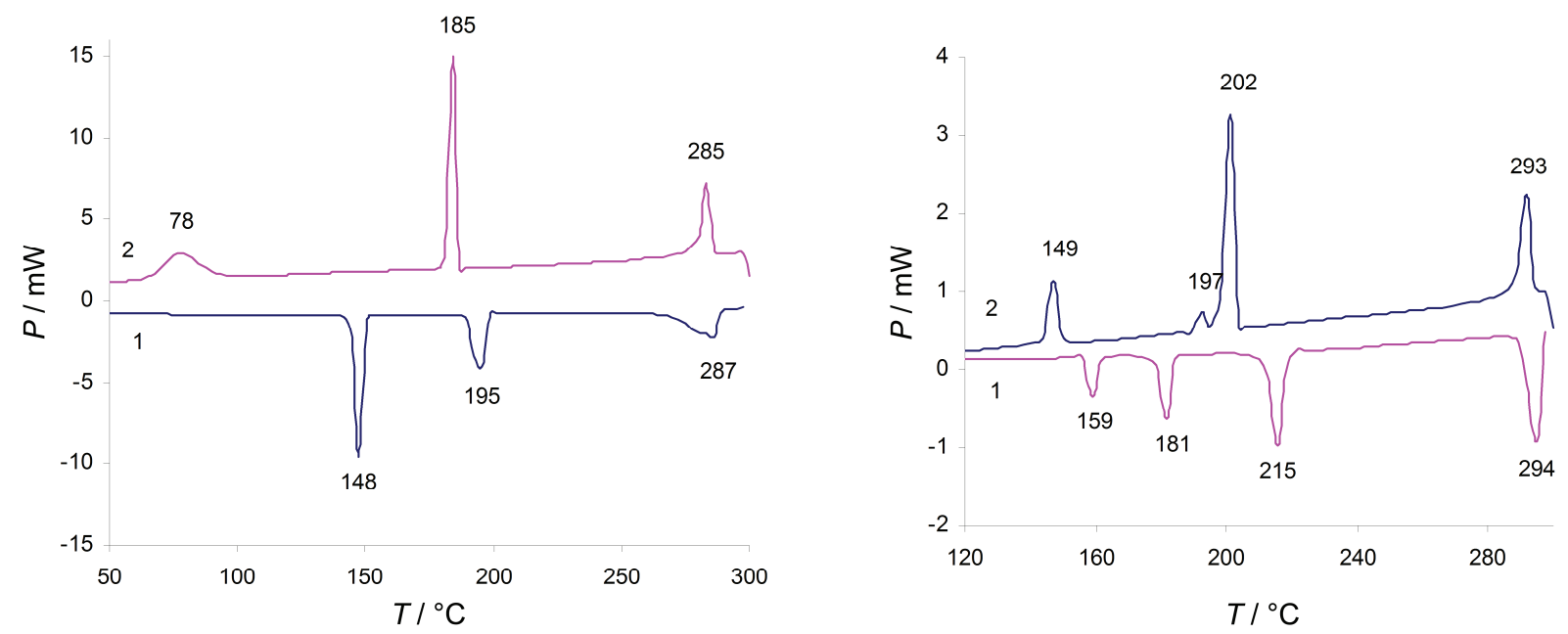

Figure 1. DSC curves: compound 5e (a): 1-first heating; 2- first cooling; 6a (b): 1-second heating; 2- first cooling.

The increasing of the length of the terminal acyloxy chain resulted in the slight decreasing of both melting and clearing temperatures for compounds $\mathbf{5 c}$ 5e. An interesting behavior showed compound $\mathbf{5 c}$ both on heating and cooling cycles. During the first heating, at $207{ }^{\circ} \mathrm{C}$ ordering in oily streaks like appearance began (Figure 3a) which changed near the isotropisation temperature to a focal conic texture (Figure 3b). On the first cooling from the isotropic liquid, lath-like and circular smectic type textures were evidenced (Figures $3 \mathrm{c}, 3 \mathrm{~d})$. The smectic ordering maintained on the second heating but the second cooling debuted with a short nematic Schlieren phase (Figure 3e) that changed to a smectic one at $263{ }^{\circ} \mathrm{C}$. 

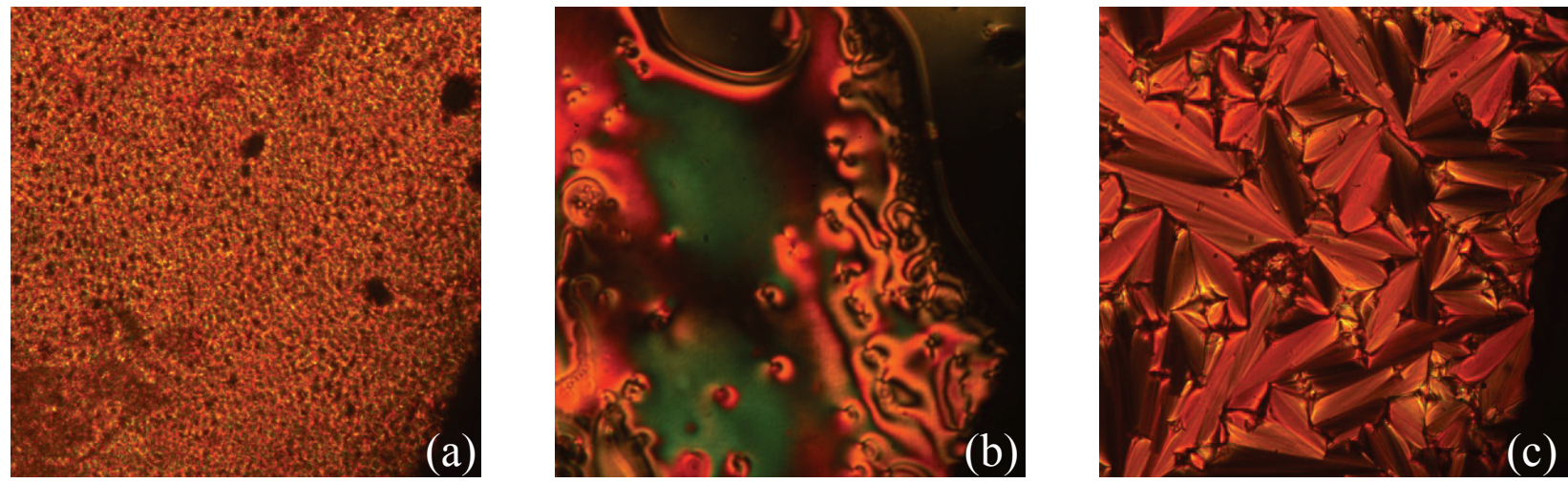

Figure 2. Optical textures: $\mathbf{5 a}, 303^{\circ} \mathrm{C}$, first heating (a); 5a, $274{ }^{\circ} \mathrm{C}$, first cooling (b); $\mathbf{5 b}, 219^{\circ} \mathrm{C}$, first cooling (c).
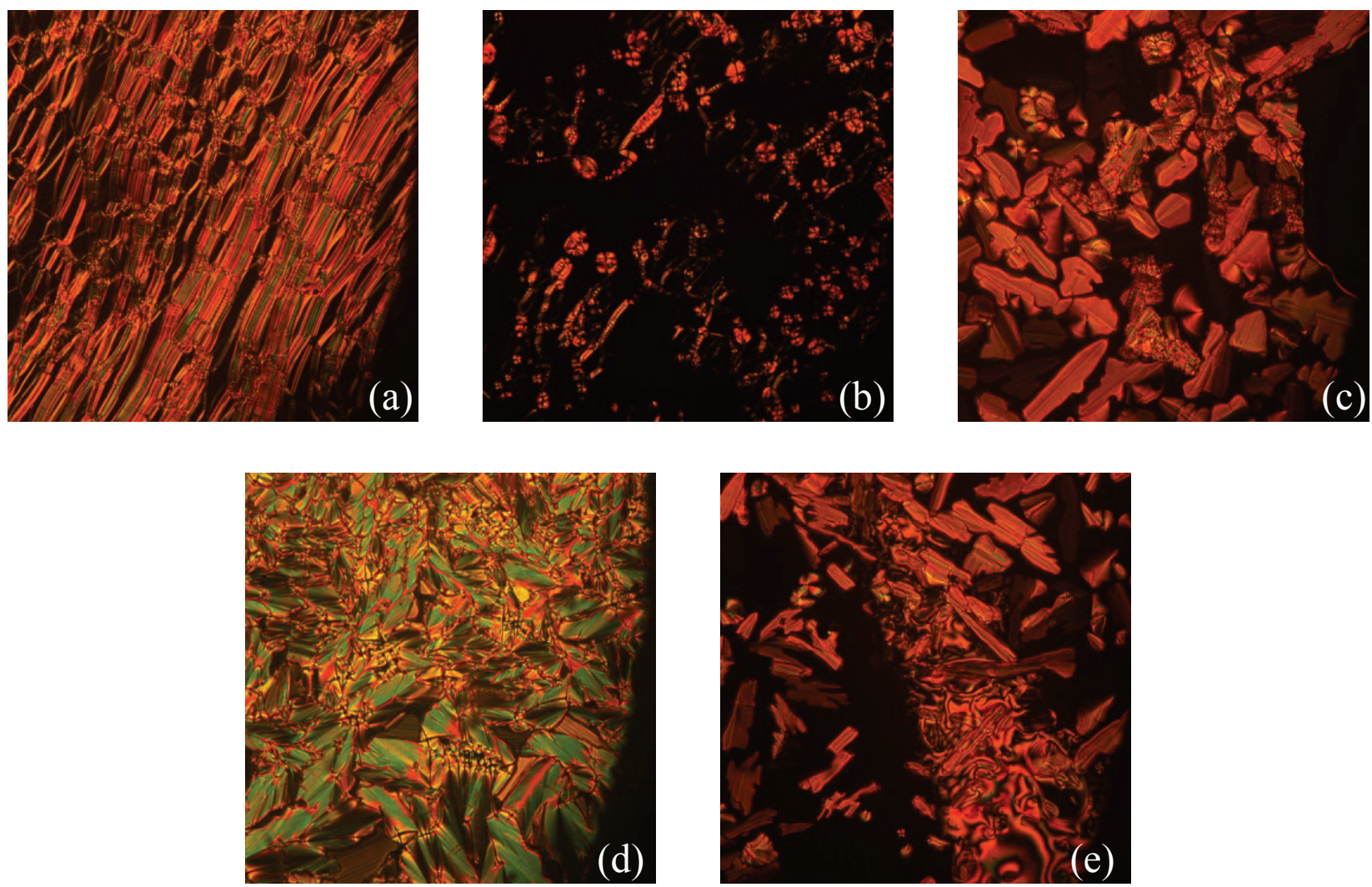

Figure 3. Optical textures of compound $\mathbf{5 c}: 234^{\circ} \mathrm{C}$, first heating (a); $282{ }^{\circ} \mathrm{C}$, first heating (b); $277{ }^{\circ} \mathrm{C}$, first cooling (c); $196{ }^{\circ} \mathrm{C}$, first cooling (d); $262{ }^{\circ} \mathrm{C}$, second cooling (e).

The last two compounds of the series showed similar thermal behavior and textures. On heating compounds $\mathbf{5 d}$ and $\mathbf{5 e}$ ordered in an unidentified mesophase as thin filaments (Figure 4a). On cooling, a nematic phase appeared for a short time accompanied by fan-shaped textures (Figure $4 b$ ) that grew up into an unidentified mesophase that maintained down for about $100{ }^{\circ} \mathrm{C}$ (Figure 4c).
The 6a-6e class of Schiff bases containing the less polar alkyloxy terminal chain evidenced a similar liquid crystalline behavior with the $\mathbf{5 a}-\mathbf{5 e}$ series. The transition temperatures have slightly lower values if compared with the $5 \mathbf{a}-\mathbf{5 e}$ series. The stability ranges of the mesophases maintains on a wide interval, between 63 and $82{ }^{\circ} \mathrm{C}$ on heating and 73 and $96^{\circ} \mathrm{C}$ on cooling. 

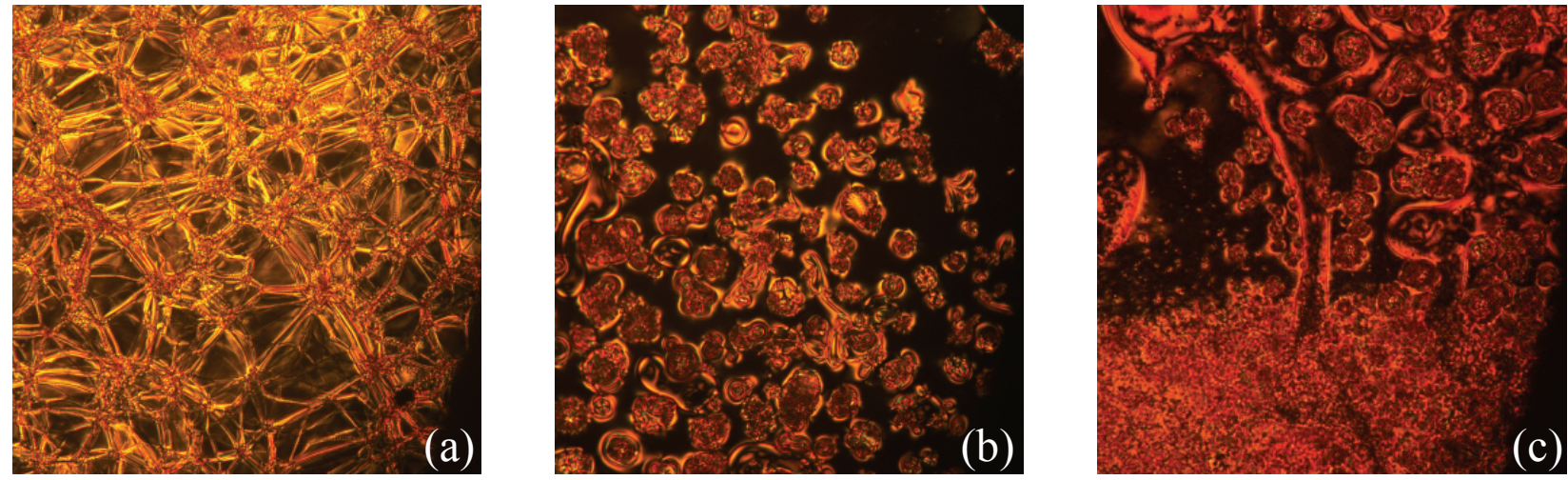

Figure 4. Optical textures: $\mathbf{5 e}, 272^{\circ} \mathrm{C}$, first heating (a); $\mathbf{5 d}, 249^{\circ} \mathrm{C}$, first cooling (b); $5 \mathbf{e}, 250{ }^{\circ} \mathrm{C}$, first cooling (c).

Figure 1b presents as an example the DSC curves for compound $\mathbf{6 a}$.

On heating, compound $\mathbf{6 a}$ evidenced an unspecific smectic texture; on cooling from the isotropic liquid a lath-like texture that developed into a dense texture (Figure 5a). On cooling, the DSC curve evidenced at $202{ }^{\circ} \mathrm{C}$ a new LC/LC transition which could not be seen on POM investigations and that maintained up to 197 ${ }^{\circ} \mathrm{C}$ when crystallization took place. Similar textures appeared for compound $\mathbf{6 b}$ (Figure 5b).
Compound 6c showed similar characteristic fanlike textures on cooling from the isotropic state which finally developed into unidentified texture that maintained up to crystallization $\left(181^{\circ} \mathrm{C}\right.$ ) (Figure $5 \mathrm{c}$ ).

Compounds 6d and 6e presented similar textures on heating but different ones during the cooling cycles. In the case of compound $\mathbf{6 d}$, a lath-like textures with stripes running across could be evidenced (Figure 6a), while for compound $\mathbf{6 e}$, around $167^{\circ} \mathrm{C}$, near the crystallization point, some circular and elongated domains
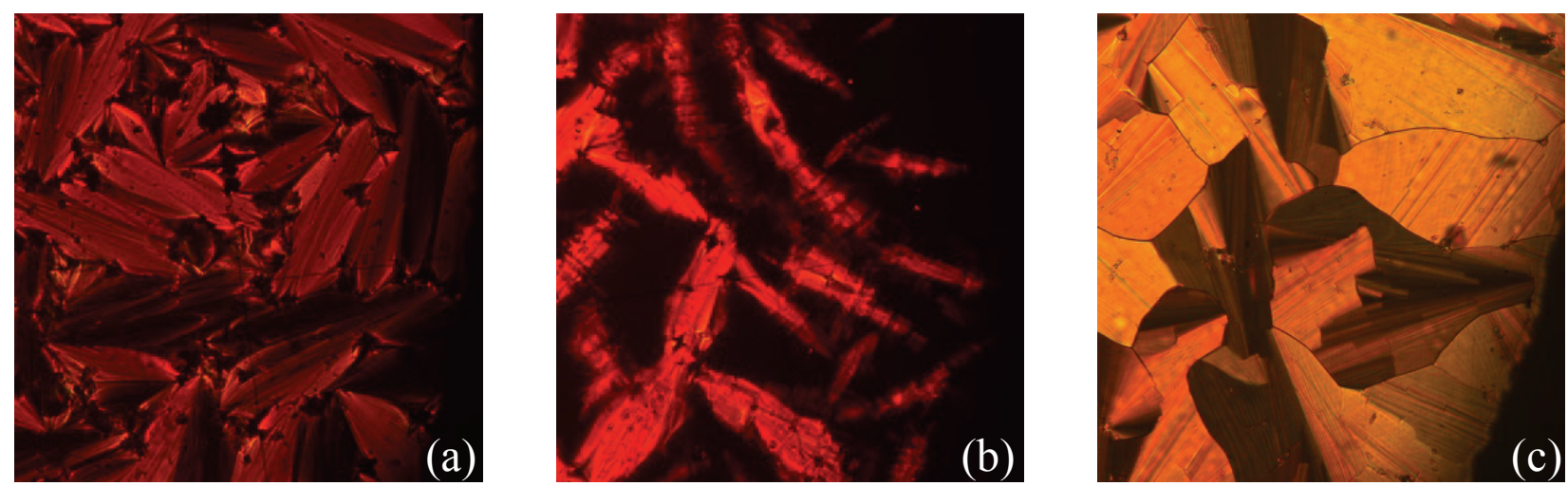

Figure 5. Optical textures: $6 \mathbf{a}, 200{ }^{\circ} \mathrm{C}$, second cooling (a); $\mathbf{6 b}, 274{ }^{\circ} \mathrm{C}$, second cooling (b); $6 \mathbf{c}, 217^{\circ} \mathrm{C}$, second cooling (c).
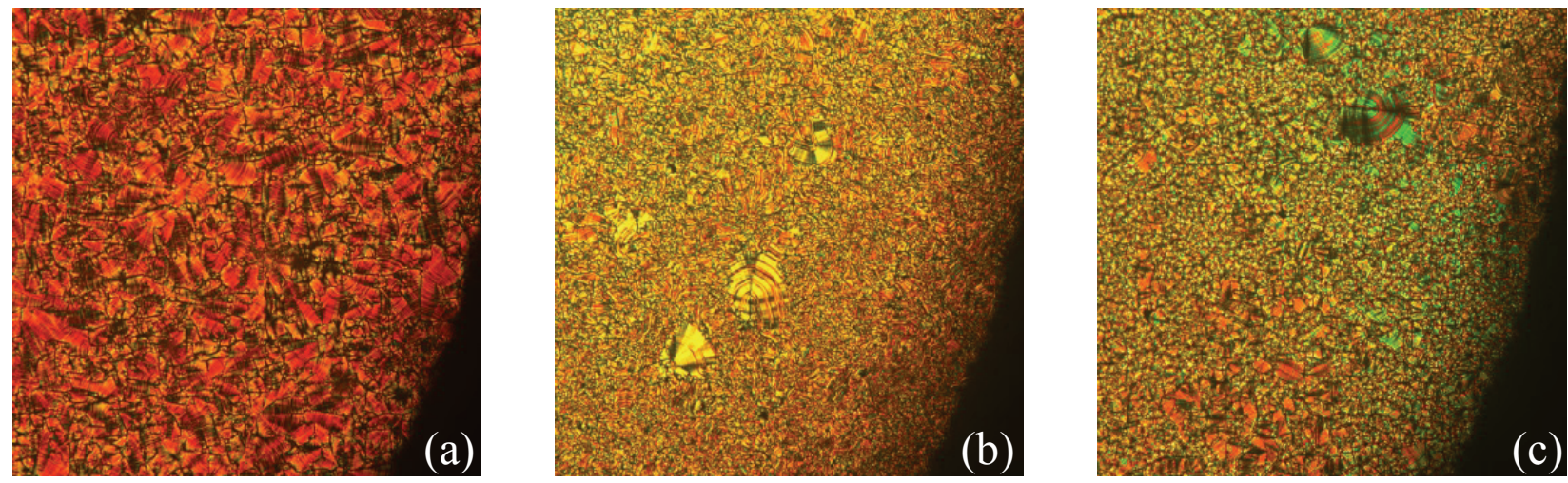

Figure 6. Optical textures: $6 \mathbf{d}, 229^{\circ} \mathrm{C}$, second cooling (a); 6e, $209^{\circ} \mathrm{C}$, second cooling (b); $6 \mathbf{e}, 167{ }^{\circ} \mathrm{C}$, second cooling (c). 


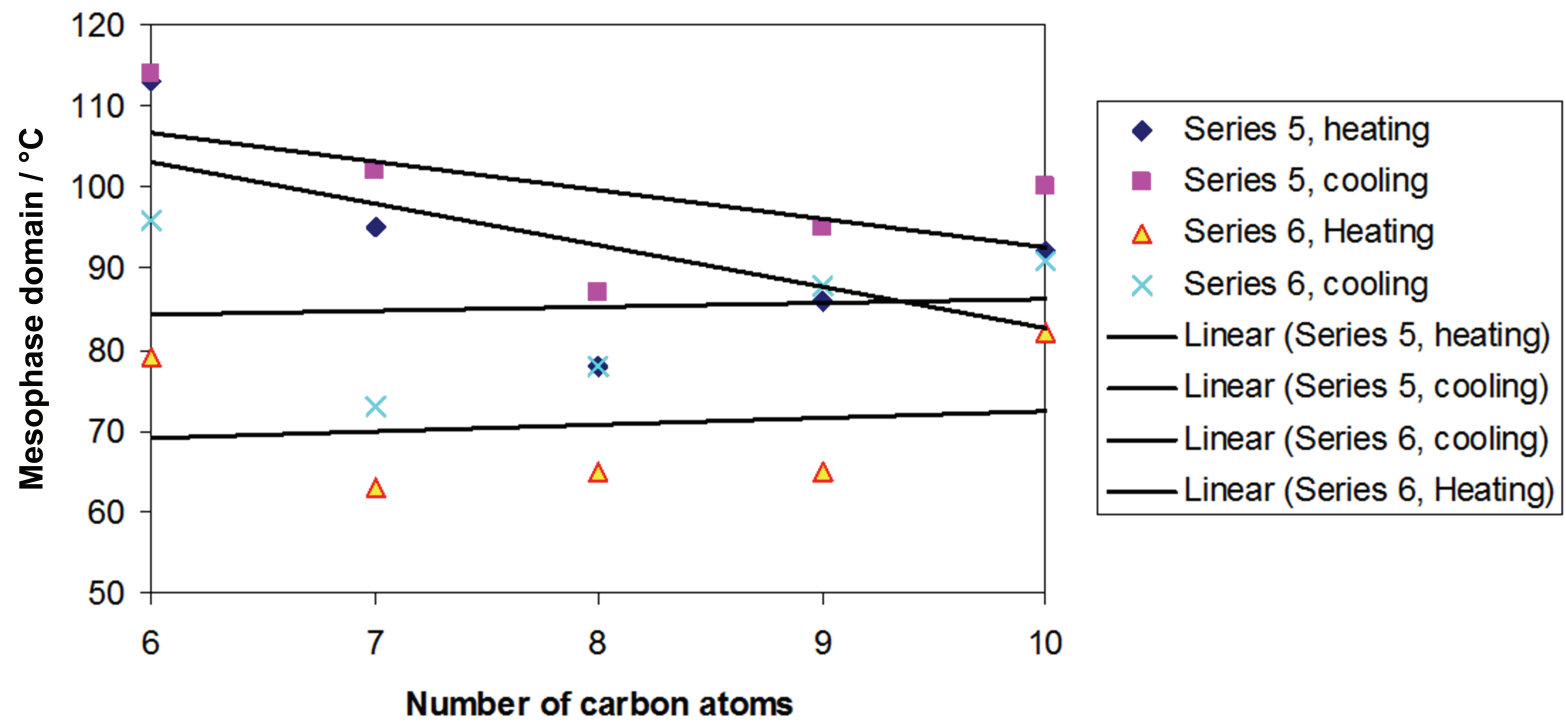

Figure 7. Domains of the mesophases stabilities for $\mathbf{5 a}-\mathbf{5 e}$ and $\mathbf{6 a}-\mathbf{6 e}$ series.

grew up from the smectic texture, which are similar with the arrangements observed for the $\mathrm{B}_{7}$ phase.

By comparing the mesophase stability for the two series of banana Schiff Bases, it has been noted that acyloxy derivatives $\mathbf{5 a}-\mathbf{5 e}$ generally showed a wider mesophase domain both on heating and cooling stages (Figure 7).

The presence of the more polar esteric linking groups between the aromatic rings and the terminal flexible chain in compounds $\mathbf{5 a - 5 e}$ slightly increases the physical interactions between the molecules in the liquid crystalline state. The mesophase stability of acyloxy compounds $\mathbf{5 a - 5 e}$ may be explained by the extended conjugated system combined with the strengthening of the lateral interactions too.

\section{CONCLUSIONS}

Two series of symmetric bent-core Schiff bases have been obtained by reacting the 1,3-phenylene bis(4-aminobenzoate) core with some 4-((4-alcanoyloxyphenyl)azo)benzaldehydes or 4-((4-alkyloxyphenyl)azo)benzaldehydes in the presence of glacial acetic acid as catalyst. The obtained compounds were characterized from the structural point of view $\left({ }^{1} \mathrm{H}\right.$ NMR, $\left.{ }^{13} \mathrm{C} \mathrm{NMR}, \mathrm{MS}\right)$. All the synthetized Schiff bases presented enantiotropic liquid crystalline properties, with a wide stability of mesophases both on heating (between 63 and $113{ }^{\circ} \mathrm{C}$ ) and cooling cycles (between 73 and $114{ }^{\circ} \mathrm{C}$ ) as evidenced by POM and DSC investigations. The observed liquid crystalline textures were mainly of smectic type.

Compounds with acyloxy terminal flexible chains presented wider mesophase stabilities both on heating and cooling cycles if compared with the alkyloxy analogues.
Acknowledgements. This work was supported by CNCS UEFISCDI (Romania), project number PNII - IDEI 356/2008.

\section{REFERENCES}

1. D. R. Link, G. Natale, R. Shao, J. E. Maclennan, N. A. Clark, E. Korblova, and D. M. Walba, Science, 278 (1997) 1924-1927

2. H. N. Shreenivasa Murthy and B. K. Sadashiva, Liq. Cryst. 31 (2004) 1347-1356.

3. G. Pelzl, S. Diele, and W. Weissflog, Adv. Mater. 11 (1999) 707-724.

4. V. Kozmik, M. Horcic, J. Svoboda, V. Novotna, and D. Pociecha, Liq. Cryst. 39 (8) (2012) 943-955.

5. G. Shanker and C. Tschierske, Tetrahedron 67 (45) (2011) 8635-8638.

6. J. Seltmann, A. Marini, B. Mennucci, S. Dey, S. Kumar, and M. Lehmann, Chem. Mater 23 (10) (2011) 2630-2636.

7. H. Takezoe, Y. Takanishi, Jpn. J. Appl. Phys. 45 (2A) (2006) 597-625.

8. R. A. Reddy and B. K. Sadashiva, Liq. Cryst. 31 (8) (2004) 1069-1081.

9. I. Dierking, Textures of Liquid Crystals, Wiley-VCH Verlag, Weinheim, 2003, pp. 167-212.

10. W. Weissflog, C. Lischka, S. Diele, G. Pelzl, and I. Wirth, Mol. Cryst. Liq. Cryst. 328 (1999) 101-109.

11. B. K. Sadashiva, V. A. Raghunathan, and R. Pratibha, Ferroelectrics 243 (2000) 249-260.

12. R. A. Reddy, B. K. Sadashiva, Liq. Cryst. 30 (2003) 1031-1050.

13. W. Weissflog, H. Nadasi, U. Dunemann, G. Pelzl, S. Diele, A. Eremin, and H. Kresse, J. Mater. Chem. 11 (11) (2001) $2748-2758$.

14. J. C. Rouillon, J. P. Marcerou, M. Laguerre, H. T. Nguyen, and M. F. Achard, J. Mater. Chem. 11 (12) (2001) 2946-2950.

15. T. Niori, T. Sekine, J. Watanabe, T. Furukawa, and H. Takezoe, J. Mater. Chem. 6 (1996) 1231-1233.

16. V. Prasad, D. S. S. Rao, and S. K. Prasad, Liq. Cryst. 28 (4) (2001) 643-646.

17. H. C. Jeong, H K. V. Le, HM. J. Gim, S.T. Hur, S. W. Choi, F. Araoka, K. Ishikawa, and H. Takezoe, J. Mater. Chem. 22 (11) (2012) 4627-4630. 
18. K. Nejati, Z. Rezvani, E. Alizadeh and R. Sammimi, J. Coord. Chem. 64 (11) (2011) 1859-1870.

19. H. Yoon, S. W. Kang, M. Lehmann, J. O. Park, M. Srinivasarao, and S. Kumar, Soft Matter 7 (19) (2011) 8770-8775.

20. E. R. Cioanca, E. L. Epure, I. Carlescu, G. Lisa, D. Wilson, N. Hurduc, and D. Scutaru, Mol. Cryst. Liq. Cryst. 537 (2011) 51-63.

21. V. Prasad, S. W. Kang, and S. Kumar, J. Mater. Chem. 13 (6) (2003) 1259-1264.
22. V. Prasad, S. W. Kang, K. A. Suresh, L. Joshi, Q. B. Wang, and S. Kumar, J. Am. Chem. Soc. 127 (49) (2005) 17224-17227.

23. C. L. Folcia, I. Alonso, J. Ortega, J. Etxebarria, I. Pintre, and M. B. Ros, Chem. Mater. 18 (19) (2006) 4617-4626.

24. L. Rahman, J. Asik, S. Kumar, and C. Tschierske, Liq. Cryst. 35 (11) (2008) 1263-1270.

25. E. R. Cioanca, C. Ciobanu, G. Lisa, and D. Scutaru, Bulletin of the Polytechnic Institute of Iaşi, Section Chemistry and Chemical Engineering 3 (2009) 35-46. 